\title{
ROADMAP TOWARDS AN
}

\section{INTEGRATED EUROPEAN}

\section{RESPONSE TO DOMESTIC}

VIOLENCE

\author{
BRANKO LOBNIKAR, ${ }^{1}$ CATHARINA VOGT $^{2} \&$ \\ JOACHIM KERSTEN ${ }^{2}$ \\ ${ }^{1}$ University of Maribor, Faculty of Criminal Justice and Security, Ljubljana, Slovenia. \\ E-mail: branko.lobnikar@um.si \\ ${ }^{2}$ German Police University, Criminology and Interdisciplinary Crime Prevention, \\ Münster, Germany. \\ E-mail: c.vogt@respectresearchgroup.org; joachim.kersten@t-online.de
}

Abstract The main goal of the IMPRODOVA project was to find ways to optimise domestic violence intervention and prevention. We found that effective cooperation of front-line responders comes from a common understanding of the problem. When trying to understand the phenomenon thoroughly, we realised that cross-national comparison of domestic violence definitions is a complex undertaking, as different countries use varied definitions. Intimate partner violence, domestic violence and family violence are used across all countries to describe the phenomenon. However, we can observe primarily gender-based definitions in all three front-line responders sectors. One of the promising findings of our analyses is that international standards are relatively well implemented in all the partner countries. For better cooperation of all stakeholders, we developed a training platform on domestic violence and supported it by analysing the possibilities of using the digital communication platform for inter-agency collaboration to address domestic violence adequately. Multidisciplinary cooperation across the sectors in risk assessment and case documentation was mentioned by many countries as a favourable objective, resulting in more dynamic and comprehensive risk assessment processes. That leads to developing a risk assessment tool - the Domestic Violence Risk Assessment Integration Module to achieve a more integrated European response to domestic violence.

Keywords: IMPRODOVA, domestic violence, Europe, police, social work, health sector, cooperation, risk assessment, training 


\section{Introduction}

The IMPRODOVA project undertook the mapping a path towards implementing the action points of the domestic violence (DV) policy framework. Analyses of various policies presently existent in project partner countries among the three frontline-responders (FLRs). In general, the IMPRODOVA project has set its research focus on the police, social services, and the health/medical sector. In the third part of this book, the authors presented research findings by individual countries involved in the project, including a description of good practices and challenges for action in future responses to domestic violence. In different ways, the country-specific differences between legal structures, policies, and national strategies against domestic violence lead to frontline responders' cooperation. These differences enable specific definitions of domestic violence and result in using a variety of risk assessment tools. In most partner countries, however, the Istanbul Convention is central to the implementation of national policies. The definition of domestic violence in the Istanbul Convention is often used as the leading definition in the country's National Action Plans, and it is based on a gender-related violence concept, mainly understood as violence against women and children. Norbert Leonhardmair, Paul Herbinger, and Marion Neunkirchner discussed this content in more detail in the first chapter of this book (see also Herbinger et al. 2020). We found that, generally, cross-national comparisons of domestic violence definitions are complex, as different countries use specific and varied definitions of domestic violence.

\section{The integrated European response to domestic violence}

Intimate partner violence, domestic violence, and family violence are the main terms used across all countries to describe the phenomena. It appears to be quite well established that violence consists of various forms and types of acts and is not restricted exclusively to physical violence. Violence includes mental, sexual, or economic aspects. Such general terms can cover multiple types of specific acts. A further gap can be demonstrated by the definition of "high-impact" domestic violence, a term not clarified by specific sub-definitions of domestic violence within national policies (Herbinger et al., 2020). 
International organisations (e.g. UN, Council of Europe) have defined a set of minimum standards that governments and service providers (SP) should achieve and implement to meet their international obligation to exercise due diligence to investigate and punish acts of violence, provide protection to victims, and prevent domestic violence. There are international standards for service providers in general and law enforcement in particular (but not specifically for NGOs or medical doctors). The foundations of the basic standards encompass confidentiality, safety, security, and respect for service users, accessibility, and availability. Support should be available free of charge, and actions taken should employ the principles of empowerment and self-determination. Service providers should be skilled and gender-sensitive, undergo ongoing training, conduct their work according to clear guidelines, protocols, and ethics codes, and, where possible, provide female staff members. Each SP should maintain the confidentiality and privacy of the victims they engage with and cooperate and coordinate with all other relevant services. It should monitor and evaluate service provision, seeking the participation of service users. The expertise of specialised NGOs should be recognised (Bradley et al., 2020).

The main findings of the IMPRODOVA analyses reveal that international standards are relatively well implemented in all the partner countries (Bradley et al., 2020; Herbinger et al., 2020). Based on the analysis, we can conclude that police have powers to enter private property and arrest and remove a perpetrator. Protection or restraining orders are available for police to tackle all forms of domestic violence. Police agencies coordinate with, and refer to, specialist support services for domestic violence victims well and that all police organisations have protocols on information sharing on DV cases with other agencies. The IMPRODOVA partners also found that some areas require special attention in the future since gaps between the international standards and the actual practice were discovered. These issues are:

a. police personnel should be trained comprehensively on aspects of domestic violence;

b. victims should be seen as soon as possible by a specially trained officer;

c. there should be at least one specialised officer per police unit, for domestic violence and for sexual violence;

d. police should proceed to risk assessment procedures supported by the timely gathering of intelligence - this intelligence should be gathered from multiple sources and seek the victim's perspective on potential threat; and 
e. police should develop and implement strategies to eliminate or reduce victims' risks.

In addition to these standards, IMPRODOVA researchers also reported deficiencies in standards related to effectively dealing with domestic violence cases: police record systems should enable identification of cases of domestic violence and permit monitoring of all measures taken, repeat victimisation, and case outcomes and police should ensure that encounters between police personnel and victims are nonjudgmental, empathetic, and supportive, and proceed in a manner that considers and prevents secondary victimisation (Bradley et al., 2020).

Comparing the case studies in the eight partner countries yields many lessons, although one sticks out: frontline responders who are specialists of domestic violence serve victims' needs better than frontline responders who are generalists. Specialists are police officers (or social workers, or medical professionals) whose job definition consists mainly of handling domestic violence cases. The generalists are police officers (or social workers, or medical professionals) who indifferently handle all cases in their work routine. The key variable, therefore, is whether victims make themselves known to specialists or generalists. Summarising the main findings, generalists will typically be less knowledgeable about domestic violence, less inclined to take non-physical violence seriously, more inclined to rely on personal discretion, and less likely to make informed and helpful referrals.

Conversely, specialists will be better trained, knowledgeable about the different types of violence, abuse and control dynamics, and the risks they entail. They are more likely to follow protocols and procedures designed to safeguard victims' interests, and they are more likely to be part of a network of professionals from other sectors who seem more likely to help the victim in their multifaceted needs. In some countries, such as Hungary, Slovenia, or Portugal, virtually all police officers on the frontline response to domestic violence are generalists. In other countries, such as Scotland and Finland, most frontline responses are made by specialists. In France, Germany, and Austria, it varies according to locations, with some places served with specialised units and others with only generalists. Beyond necessary discussions on territorial equality, the pattern that the IMPRODOVA team has identified about specialists and generalists proves true both in cross-country comparison and within- 
country differentiation. In all countries, the basic steps of a domestic violence case are the same (Bradley et al., 2020).

The gap analysis of DV data provisions revealed that data provisions for IMPRODOVA partner countries do not enable a direct or easy comparison of the results of national victimisation surveys, nor do they support a sophisticated secondary analysis including such comparisons (Fagerlund \& Houtsonen, 2019, 2021). The data provisions are heterogeneous in their sampling and data collection methodology, representability, definitions of DV, the inclusion of questions about reporting to police and other authorities, the consequences (seriousness) of violence, and their relation to national legislation. The Istanbul Convention requires that data collection and research be briefly presented in the convention, but based on the gap analysis of data sources, we conclude that, unfortunately, nationally representative data, gathered at regular intervals and including all forms of violence covered by the convention, are not available. According to IMPRODOVA analysis (Fagerlund \& Houtsonen, 2019, 2021), the police data sources seem promising and systematic in the broad picture of data provision. Police data appear to be systematically available, at least in a form that allows statistical reporting and secondary analysis. Legal differences in criminal codes may be taken into account in a way that may enable comparative analyses with limitations based on crime statistics. However, these data sources in themselves are not without problems. In addition to differences in legislation affecting practical police work, the police forces are organised differently in different countries. Most country reports also included assessments of considerable deficiency concerning the quality of police data. The data may be more illustrative about police actions, such as reporting and the use of data systems, than the actual phenomenon of domestic violence itself. From other data concerning high-impact domestic violence (HIDV), homicide data seem most promising. It is somewhat standardised compared to other data and is available generally from all IMPRODOVA partner countries. However, analysis of DV-related homicide specifically may include similar issues found in other crime data, and this should be examined more thoroughly in future IMPRODOVA research publications focusing on secondary analysis. Homicide data could also provide information usable in constructing a risk assessment tool. 
Analysing data harmonisation and consolidation, we found that domestic violence data is gathered across various registers and contexts. In looking across these sources, far from being simple, domestic violence data are marked by its variable categories, definitions, and measures, the fluidity of such features to their temporal and spatial contexts, and in some cases, the ambiguity of data categorisations and the meaning. This proves true in the internal efforts of IMPRODOVA partners to compile a comprehensive, comparative picture of country data, and it is also apparent in varying categories and definitions specified in European data requirements outlined in the Istanbul Convention, EIGE administrative data recommendations (2019), and surveys such as FRA (2014). In addition, current debates related to survey data further underscore the contested nature of data measurements and definitions. The resulting question might well be: to what extent can data be meaningfully harmonised, consolidated and compared? As a result of the analyses, the following recommendations for improving data practices were provided (Burman, Brooks-Hay, \& Bradley, 2020, pp. 20-23):

1. Efforts to harmonise data should be underpinned by a clear understanding of the aims, meaning and feasibility of 'data harmonisation' and 'consolidation' concerning different data sources.

Key to this understanding is clarity about the purpose of data harmonisation and how data will be gathered and used. Data harmonisation may occur within countries (across agencies such as police, prosecutors, health, and housing) or across countries for (a) comparability or (b) conceptual alignment (based on research evidence and knowledge about domestic violence and forming the basis of minimum standards/indicators). Surveys are best placed to elicit insights directly from victims and facilitate comparability across countries, while administrative data gathering benefits from conceptual alignment across agencies and countries on key indicators such as age, sex, and the relationship between victim and perpetrator. It should be noted that a harmonised EU definition of domestic violence is likely to be reduced to high impact domestic violence (HIDV), which privileges physical violence. This could effectively obscure all other forms of domestic violence and, in turn, have adverse implications for national interventions.

2. Measuring the extent of domestic violence reported to the police in terms of the numbers of victims, perpetrators, and offences, as recommended by EIGE (2019), should be a minimum standard for police data gathering. These data 
work to raise awareness of the scale of the problem, monitor change over time, and inform the allocation of adequate resources to tackle the problem. As it currently stands, police data on the number of offences are more readily available than the number of victims and perpetrators.

3. Data should be recorded on police action taken in response to acts (incidents) reported as domestic violence, including those incidents not later recorded as criminal offences. This measure provides essential information about incidents coming to the attention of the police and how the police respond to incidents reported to them.

4. Data on types of abuse (e.g. physical, sexual, psychological, and economic) should be priority categories for survey data collection. The collation of this data within surveys should be prioritised and recognised as complementary to administrative data due to the limitations of administrative data in relation to these variables. Indicators relating to types of abuse (and their seriousness) are populated using crime codes as a proxy, yet there are notable limitations of this approach since some types of abuse (e.g. economic and psychological) and are not well recognised or defined in criminal codes.

5. As identified by the Istanbul Convention, recording the sex of the victim and perpetrator and assessing the relationship between them should be a minimum standard for police and survey data gathering. In addition to collecting data on sex, recording the gender identity of victims and perpetrators would be a further step towards inclusivity. These data are crucial to understanding the gendered dynamics of domestic violence and, in particular, intimate partner violence. Data should be able to be disaggregated in order to be of optimum use for FLRs.

6. Where domestic violence data are gathered on violence/abuse perpetrated in a range of family relationships or a domestic unit (as per the definition of domestic violence adopted by the Istanbul Convention), there should be a clear delineation of these relationship categories, and this must include categories for violence/abuse perpetrated by intimate partners and/or ex-partners. Family and other close relationships form a context of violence in which power relations and other factors relevant to the dynamics of violence contribute to the eminently damaging nature thereof in these relationships while simultaneously making it particularly difficult for FLRs to identify cases and intervene. This can happen, e.g., in the case of parental violence against their children, violence perpetrated by adult children against their elderly parents, or violence perpetrated by affinal kin. However, violence perpetrated by partners or ex- 
partners has a distinctive dynamic and should be clearly delineated in gathering and reporting data.

7. Data should be gathered regarding repeat offences and victimisation, and the impact of domestic violence and abuse on victims is a minimum standard for survey data gathering. This data is central to understanding the gendered, ongoing, and coercive nature of domestic violence. These dimensions are important to operational police responses though they are difficult to record consistently within police administrative data, hence the importance of capturing this information directly from victims within surveys.

8. Consideration should be given to how cases reported to the police can be tracked through the criminal justice system (e.g., using unique identifiers for individual cases). The capacity to track cases throughout the criminal justice system will provide the basis for an in-depth understanding of individual cases as they progress through the system. While this recommendation extends beyond police data gathering, this process begins with the police. With victim privacy and data sharing concerns in mind, the use of a unique identifier should be strictly in relation to case tracking, and it should not be shared with agencies outside of the criminal justice system (e.g. health and housing). Unique identifiers pose a threat to privacy and the rights of the accused, and so, while they have undoubted advantages, any implementation needs to consider data infringement risks very carefully.

9. NGOs, social work services, and medical services are important sources of data and can provide information about the incidence and impact of domestic violence across different populations. Consideration should be given to using the definition of domestic violence adopted by the Istanbul Convention in data recording and the utilisation of de-identified and aggregated health or social service data to respond to domestic violence at both individual and community levels. Domestic violence victims' health and social care needs can inform measures that can improve a victim's quality of life and prevent future abuse; however, there are significant issues of confidentiality that must be respected in relation to health and social care data.

10. The needs and demands placed upon FLRs should be a key consideration for development. Adequate support, resources, and GDPR knowledge should be provided for FLRs as they progress their casework and data-recording responsibilities. To minimise the data-gathering burden placed upon FLRs such 
as the police, it should be recognised that surveys are best placed to elicit data from victims on issues such as impact, nature and extent of the abuse.

11. The unidirectional flow of data from FLRs to data gathering systems should be addressed by ensuring that FLRs are data recipients rather than providers. 'Closing the loop' for FLRs will allow FLRs to locate and understand their actions in relation to managing and mitigating domestic violence.

12. Administrative and survey analyses should be made available to the public (and FLRs) and should be made accessible to them. National (anonymised) domestic violence data should be publicly available without request. Accessibility should also be considered in relation to the format and presentation of statistical information.

13. Raw data should be made available for further analyses. Making (anonymised) raw data available to relevant agencies and researchers facilitate analytical insight beyond the headline analyses published as standard and enhances the utility of the data gathered.

14. The EU and the Member States should promote and fund surveys that can be repeated every few years to measure developments over time. This recommendation concurs with the FRA (2014) recommendation on this issue, and its adoption would signify a concerted effort to uncover information on the extent and nature of domestic violence.

15. Alternative methods of gathering and utilising data about the "bigger picture" of domestic violence should be considered, in addition to the use of administrative and conventional survey data. (Burman, Brooks-Hay, \& Bradley, 2020)

Part of the IMPRODOVA project included the development of a risk assessment tool - Domestic Violence Risk Assessment Integration Module - RAIMO, which is described in detail in the chapter by Marianne Mela and Jarmo Houtsonen in this book. Concerning risk assessment, problematic aspects emerged in all sectors (Hera \& Szegö, 2020). Many countries highlighted the rigidity of existing formal risk assessment tools. Some countries have adopted Multi-Agency Risk Assessment Conferences (MARAC). A wide range of statutory and non-statutory agencies participate and share information in these conferences to develop safety plans for high-risk victims of domestic violence. In addition to using MARACs, Scotland has implemented Multi-Agency Tasking and Coordination groups (MATACs) to target and identify repeat offenders of domestic abuse. The MARAC project in Austria was 
negatively evaluated by the participating organisations, as the cooperation between FLRs was ineffective in the context of these conferences. This can, among other variables, be traced back to the strength of data protection regulations, which make it challenging to exchange sensitive information among different FLRs. In this regard, Scotland can be mentioned as an outstanding example for including specialist domestic abuse courts as additional key actors in domestic violence cases. The Scottish courts are unique in their emphasis on the significance of multi-agency cooperation between the police, prosecution, and specialist domestic abuse service providers and reflect the policy aim of improving the coordination of information across criminal justice agencies (Hera \& Szegő, 2020). The IMPRODOVA researchers also reported that some professionals do not condone the use of checklists since, in their experience, such tools do not reflect the particulars of domestic violence incidents. Those tools are seen as too rigid and not sensitive enough to fit individual cases, resulting in false assessment and the negligence of risk situations that do not "fit into the boxes". Thereby, many professionals across the countries argued that formalised tools have to be accompanied by comprehensive and regular professional training and personal expertise.

As a favourable objective, which might result in more dynamic and comprehensive risk assessment processes (Delpeuch \& Bonnet, 2020; Machado et al., 2021; Vogt, 2020; also cf. the chapter by Thierry Delpeuch and François Bonnet in this book), multidisciplinary cooperation across the sectors in risk assessment and case documentation was mentioned by many countries in all sectors. Unified risk assessment and case documentation protocols are the preconditions of such an endeavour. Cross-referenced analysis of the 18 case studies shows that the consolidation of a partnership against domestic violence depends on many factors that have nothing to do with the partnership organisation's design and management. By consolidation, IMPRODOVA researchers mean the institutionalisation and systematic use of working procedures by which partnership bodies and partnership relays contribute and act together in an integrated manner to provide efficient partnership services. These factors include:

a. The existence of a legal framework or public policy that encourages or even enforces partner organisations to engage in the partnership and consider it a priority. These incentives can be negative (regulatory obligation, hierarchical 
order, etc.) or positive (granting of subsidies, allocating additional resources, etc.).

b. Increased social, political, and media pressure to strengthen the efforts against DV. These pressures are often linked to public opinion cases, advocacy, or the adoption of international standards.

c. Strong involvement of institutional entrepreneurs and change agents in the design and promotion of the partnership mechanism and their ability to build alliances with influential partner organisations' members (Vogt, 2020).

d. Securing political support, especially from local authorities.

e. Reference to models applied elsewhere - within the national territory or abroad - that are already acknowledged as "good practices". Such recognition is rarely linked to the availability of rigorous evaluations of "good practices" effectiveness. It most often results from the notoriety of the institutions that have pioneered the practice or are working to disseminate it.

If we want to achieve optimised domestic violence prevention and response, we found that the most effective cooperation of first-line responders comes from a common understanding of the problem. For this reason, we developed a training platform on domestic violence (https://training.improdova.eu/en/) and supported it by analysing the possibilities of using the digital communication platform for interagency collaboration to manage domestic abuse (Pfleiderer \& Juszczyk, 2021). In addition to the domestic-violence risk-assessment integration module (RAIMO), the training platform is probably one of the more relevant and applicable results of the IMPRODOVA project, as it includes all first responders in training, whom we found to have a significant impact on the final success of responding to domestic violence. The importance of training is thoroughly presented by Bettina Pfleiderer and Paulina Juszczyk in this book.

\section{Conclusion}

During the course of the IMPRODOVA project, we presented and published findings, along with providing recommendations addressing multiple audiences and stakeholders. Through years of research, we believe that we have added our pebble to the mosaic of improved and embedded collaborative working between police, other frontline and first-responder agencies, and pertinent stakeholders. As already mentioned, one of the key results of our work is optimised domestic violence 
prevention and response through innovative, gender-sensitive training and education, challenging the traditional masculine-oriented cultures and attitudes within the police. What we find particularly important is that the training platform will be useful in the future, that it is freely accessible, and that it creates the conditions for good cooperation between first responders to domestic violence. We are confident that development, validation, and embedding of common riskassessment practices, combined with increased understanding and awareness, cultural and attitudinal shifts, and openness to collaborative working, will lead to increased victim protection, occupational safety, more efficient use of resources, better cooperation between agencies, and an improved working climate for first responders. So, in the longer term, the improvements put in place by IMPRODOVA will likely raise awareness of the problematic nature of domestic violence among broader groups of citizens and contribute to improving gender equity.

\section{References}

Bradley, L.; Brooks-Hayes, O.; Burman, M.; Bonnet, F.; Cuillerdier, F. Delpeuch, T.; Felgueiras, S.; Giljohann, S.; Hera, G.; Herbinger, P; Houtsonen, J.; Jaffré, J.M.; Jereb, K.; Kersten, J. Leonhardmair, N.; Limonier, C.; Lobnikar, B:, Machado, P.; Mela, M:, Morgado, S.; Neunkirchner, M.; Nipuli, S. October, M.; Pais, L.; Pfleiderer, B.; Richter, L.; Slak, B.; Szegő, D. Vassileva, M. (2020). Country Reports and Cross-National Comparison on the Implementation of International Norms and National Best Practices of Frontline Responders. IMPRODOVA Consortium - Deutsche Hochschule der Polizei.

https://www.improdova.eu/pdf/IMPRODOVA_D2.2_Norms_and_Best_Practices_of_Fro ntline_Responders.pdf?m=1585673378\&

Burman, M., Brooks-Hay, O. \& Bradley, L. (2020). Recommendations for data harmonisation and consolidation. IMPRODOVA Consortium, Deutsche Hochschule der Polizei. https://www.improdova.eu/pdf/Improdova_D3.2_Recommendations_Data_Harmonisatio n_Consolidation.pdf?m $=1591377139 \&$

Delpeuch T. \& Bonnet F. (2020). Identifying Gaps and Bridges of Intra- and Inter-Agency Cooperation. IMPRODOVA Consortium, Deutsche Hochschule der Polizei. https://www.improdova.eu/pdf/IMPRODOVA_D2.4_Gaps_and_Bridges_of_Intra_and_Interagency_Cooperation.pdf?m $=1585673383 \&$

Fagerlund, M. \& Houtsonen, J. (2019). Map of Available Data on Domestic Violence. IMPRODOVA Consortium - Deutsche Hochschule der Polizei. https://www.improdova.eu/pdf/Map-ofAvailable-Data-on-Domestic-Violence.pdf?m $=1555573584 \&$

Fagerlund, M., \& Houtsonen, J. (2021). Status Quo of National Data Sources Concerning Domestic Violence Across Eight European Countries. European Law Enforcement Research Bulletin, 21, 101 - 120. https://bulletin.cepol.europa.eu/index.php/bulletin/article/view/419

Hera, G. \& Szegő, D. (2020). Country Reports and Cross-National Comparison on the Risk Assessment Tools and Case Documentation used by Frontline Responders. IMPRODOVA Consortium, Deutsche Hochschule der Polizei.

https://www.improdova.eu/pdf/IMPRODOVA_D2.3_Risk_Assessment_Tools_and_Case _Documentation_of_Frontline_Responders.pdf?m $=1585673380 \&$ 
Herbinger, P. L., Neunkirchner, M., \& Leonhardmair, N. (2020). European legislation to fight domestic violence. European Law Enforcement Research Bulletin, 20, 141154. http://bulletin.cepol.europa.eu/index.php/bulletin/article/view/415/339

Houtsonen, J. (2020). Policing Domestic Violence: Strategy, Competence, Training. European Law Enforcement Research Bulletin, 19, 135-155.

https://bulletin.cepol.europa.eu/index.php/bulletin/article/view/417/318

Machado, P., Pais, L., Morgado, S., \& Felgueiras, S. (2021). An Inter-Organisational Response to Domestic Violence. European Law Enforcement Research Bulletin, 21, 121-139. https://bulletin.cepol.europa.eu/index.php/bulletin/article/view/418

Pfleiderer, B., \& Juszczyk, P. (2021). European Online Training Platform on Domestic Violence Improving Frontline Responses to Domestic Violence and Sexual Assault; Pp 16-23. In: Megh, M. (2021). PET Prevention, Examination and Treatment of Domestic Violence and Sexual Assault Cases. 1st edition 2021. CBS Publishers \& Distributors.

Vogt, C. (2020). Interagency Cooperation. European Law Enforcement Research Bulletin, 19, 153-163. http://bulletin.cepol.europa.eu/index.php/bulletin/article/view/412/323 
